\title{
Prediction of Tumor Grade and Nodal Status in Oropharyngeal and Oral Cavity Squamous-cell Carcinoma Using a Radiomic Approach
}

\author{
VALERIA ROMEO ${ }^{1 *}$, RENATO CUOCOLO ${ }^{1 *}$, CARLO RICCIARDI ${ }^{1}$, LORENZO UGGA $^{1}$, SIRIO COCOZZA $^{1}$, \\ FRANCESCO VERDE $^{1}$, ARNALDO STANZIONE ${ }^{1}$, VIRGINIA NAPOLITANO ${ }^{2}$, DANIELA RUSSO ${ }^{2}$, \\ GIOVANNI IMPROTA $^{3}$, ANDREA ELEFANTE ${ }^{1}$, STEFANIA STAIBANO ${ }^{2}$ and ARTURO BRUNETTI ${ }^{1}$ \\ ${ }^{1}$ Department of Advanced Biomedical Sciences, Diagnostic Imaging section, \\ University of Naples "Federico II", Naples, Italy; \\ ${ }^{2}$ Department of Advanced Biomedical Sciences, Pathology section, \\ Head and Neck research group (GIPaTeC, SIAPEC), University of Naples "Federico II", Naples, Italy; \\ ${ }^{3}$ Department of Public Health, University of Naples "Federico II", Naples, Italy
}

\begin{abstract}
Background/Aim: To investigate whether a radiomic machine learning (ML) approach employing texture-analysis (TA) features extracted from primary tumor lesions (PTLs) is able to predict tumor grade (TG) and nodal status (NS) in patients with oropharyngeal $(O P)$ and oral cavity (OC) squamous-cell carcinoma (SCC). Patients and Methods: Contrast-enhanced CT images of 40 patients with $O P$ and $O C$ SCC were post-processed to extract TA features from PTLs. A feature selection method and different $M L$ algorithms were applied to find the most accurate subset of features to predict TG and NS. Results: For the prediction of $T G$, the best accuracy (92.9\%) was achieved by Naïve Bayes $(N B)$, bagging of $N B$ and $K$ Nearest Neighbor $(K N N)$. For the prediction of NS, J48, NB, bagging of NB and boosting of $J 48$ overcame the accuracy of $90 \%$. Conclusion: A radiomic ML approach applied to PTLs is able to predict TG and NS in patients with $O C$ and $O P$ SCC.
\end{abstract}

Cancers of the oral cavity (OC) and oropharynx (OP) squamous cell carcinoma (SCC) arise from the uppermost part of the digestive tract with a reported incidence of $2.1 \%$ and

\footnotetext{
*These Authors contributed equally to this study.

Correspondence to: Valeria Romeo, MD, Ph.D., ORCID ID: 00000002-1603-6396, Department of Advanced Biomedical Sciences, University of Naples "Federico II", Via S. Pansini, 5, 80131 Naples, Italy. Tel: +390817462039 (3560), Fax: +39 0815457081, e-mail: valeria.romeo@unina.it
}

Key Words: Head and neck squamous cell carcinoma, computed tomography, texture analysis, machine learning.
$1 \%$, respectively (1), causing significant mortality and morbidity despite the fact that clinical advances enable their early diagnosis and treatment. Well recognized risk factors of cancer of the OC and OP are smoking, alcohol and sexual behavior (2). Once detected by clinical and/or endoscopic examination, imaging is required for tumor staging in order to establish the most appropriate treatment strategy. Surgery is the first choice at early stages while a combination of chemotherapy and radiotherapy is recommended in advanced stages (3), depending on tumor size and the presence of metastatic lymph-nodes. The surgical approach consists of primary tumor resection and neck dissection. In particular, the type of neck dissection (selective $v s$. comprehensive) is defined on the basis of pre-operative $\mathrm{N}$ stage (4). The presence of metastatic lymph-nodes is considered the single most important negative predicting factor, reducing, when present, overall 5-year survival by $50 \%$. Along with nodal status, tumor grade is reported as an independent predictor of distant metastases, also associated with recurrence after surgery (5) and affecting overall survival (6). Both nodal status and tumor grade play a role in determining patients who may benefit from systemic treatment (7). In this setting, additionally to ultrasound, CT and MR, advanced imaging techniques (i.e. perfusion and diffusion weighted imaging), have been employed to identify non-enlarged, positive lymph-nodes in head and neck cancer patients (8-10). However, even if promising, the role of these new modalities has still to be established. Texture analysis (TA) is a post-processing imaging technique that has the potential to provide data on tumor biology by the extraction of quantitative features reflecting image heterogeneity in terms of pixel gray level value distribution. TA-derived features are categorized as follows: 1) first-order texture features (histograms of pixel 
intensity levels), reflecting average pixel values and their distribution; 2) second-order texture features (gray-level cooccurrence matrices) which describe a pixel joint probability function within the region of interest; 3 ) higher-order (runlength matrices) texture features, reflecting value distribution of neighboring pixel groups. Differently from first order, second and higher-order features also contain spatial information about gray level value distribution. Machine learning is an application of artificial intelligence that can develop predictive algorithms using texture features and could be applied in different fields, from diagnosis to the prediction of patients' prognosis and/or response to a specific treatment, towards a "personalized medicine" (11-14).

When previously employed in head and neck cancer imaging, TA and machine learning applied to CT, PET or MR images have been proven to be reliable in predicting Human Papilloma Virus (HPV) status $(15,16)$, p53 mutation (17), local treatment (18) as well as patients failure (19) and overall survival (20). In this respect, we performed a machine learning analysis using CT-derived TA features in patients with histologically proven OC and OP SCC, aiming to assess whether this approach may predict tumor grade and nodal status.

\section{Patients and methods}

This retrospective study was approved by the local Institutional Review Board and Ethical Committee and written informed consent was waived.

Patient population. From our database, all consecutive CT examinations performed between January 2014 and December 2016 for pre-operative staging of patients with clinical diagnosis of head and neck carcinoma, prior to biopsy, were selected $(n=116)$. Inclusion criteria were: $>18$ year-old patients with OC and OP SCC who underwent a contrast-enhanced CT scan at our Institution, and subsequently underwent surgical excision and for whom histological report was available $(n=58)$. Exclusion criteria were the following: patients with tumor recurrence $(\mathrm{n}=8)$; $\mathrm{CT}$ images affected by motion or beam hardening artifacts by dental implants $(n=4)$; CT examinations in which tumor lesions were not clearly detectable $(n=6)$. Based on these criteria, 40 patients $(21 \mathrm{M}$, mean age $=69 \pm 14$ years $)$, of which 33 with OC and 7 with OP carcinoma, were finally included. At histopathological examination, 13 patients were classified as G2 and 27 as G3 lesion. In 19 cases, no metastatic cervical lymph-nodes were found, while 21 patients presented with metastatic cervical nodal involvement. Histological features of patient population along with the employed surgical approach are reported in Table I. The flow-chart for patient selection is reported in Figure 1.

Pathology. Formalin-fixed, paraffin-embedded tissue blocks of OC and OP cases were retrieved from the archives of our institution. For each case, a block of tissue fixed in formalin and embedded in paraffin representative of the tumour was selected and used it to obtain serial sections. One section was stained with haematoxylin/eosin to confirm the initial diagnosis (Figure 2); the remaining were used for immunohistochemical investigation. The histotype was SCC for all
Table I. Clinical and histological features of patient population

\begin{tabular}{|c|c|c|c|c|}
\hline Patient & Site & p16 status & Surgery & pTNM \\
\hline 1 & OC SCC & - & $\mathrm{PTR} \pm \mathrm{BND}$ & pT4G2N0 \\
\hline 2 & OP SCC & neg & $\mathrm{PTR} \pm \mathrm{IND}$ & pT2G3N0 \\
\hline 3 & OC SCC & - & $\mathrm{PTR} \pm \mathrm{BND}$ & pT4G3N2b \\
\hline 4 & OC SCC & - & $\mathrm{PTR} \pm \mathrm{BND}$ & pT4aG3N2b \\
\hline 5 & OC SCC & - & $\mathrm{PTR} \pm \mathrm{IND}$ & pT2G2N0 \\
\hline 6 & OC SCC & - & $\mathrm{PTR} \pm \mathrm{IND}$ & pT2G3N0 \\
\hline 7 & OP SCC & neg & $\mathrm{PTR} \pm \mathrm{IND}$ & pT2G3N2b \\
\hline 8 & OC SCC & - & $\mathrm{PTR} \pm \mathrm{IND}$ & pT2G3N2b \\
\hline 9 & OP SCC & neg & $\mathrm{PTR} \pm \mathrm{BND}$ & pT3G3N0 \\
\hline 10 & OC SCC & - & $\mathrm{PTR} \pm \mathrm{BND}$ & pT4G3N2a \\
\hline 11 & OP SCC & neg & $\mathrm{PTR} \pm \mathrm{IND}$ & pT2G3N1 \\
\hline 12 & OC SCC & - & $\mathrm{PTR} \pm \mathrm{BND}$ & pT3G2N0 \\
\hline 13 & OC SCC & - & $\mathrm{PTR} \pm \mathrm{IND}$ & pT2G3N1 \\
\hline 14 & OC SCC & - & $\mathrm{PTR} \pm \mathrm{BND}$ & pT4G3N0 \\
\hline 15 & OC SCC & - & $\mathrm{PTR} \pm \mathrm{SLNB}$ & pT1G3N0 \\
\hline 16 & OC SCC & - & $\mathrm{PTR} \pm \mathrm{BND}$ & pT4aG2N1 \\
\hline 17 & OC SCC & - & $\mathrm{PTR} \pm \mathrm{BND}$ & pT3G2N0 \\
\hline 18 & OC SCC & - & $\mathrm{PTR} \pm \mathrm{IND}$ & pT2G3N0 \\
\hline 19 & OC SCC & - & $\mathrm{PTR} \pm \mathrm{BND}$ & pT3G2N0 \\
\hline 20 & OP SCC & neg & $\mathrm{PTR} \pm \mathrm{BND}$ & pT4G3N1 \\
\hline 21 & OC SCC & - & $\mathrm{PTR} \pm \mathrm{IND}$ & pT2G2N1 \\
\hline 22 & OC SCC & - & $\mathrm{PTR} \pm \mathrm{BND}$ & cT4G1N0 \\
\hline 23 & OC SCC & - & $\mathrm{PTR} \pm \mathrm{IND}$ & pT2G3N1 \\
\hline 24 & OC SCC & - & $\mathrm{PTR} \pm \mathrm{SLNB}$ & pT1G1N0 \\
\hline 25 & OC SCC & - & $\mathrm{PTR} \pm \mathrm{BND}$ & pT4G3N0 \\
\hline 26 & OP SCC & neg & $\mathrm{PTR} \pm \mathrm{IND}$ & T2G3N0 \\
\hline 27 & OC SCC & - & $\mathrm{PTR} \pm \mathrm{IND}$ & pT2G3N2b \\
\hline 28 & OC SCC & - & $\mathrm{PTR} \pm \mathrm{BND}$ & $\mathrm{pT} 1 \mathrm{G} 2 \mathrm{~N} 2 \mathrm{c}$ \\
\hline 29 & OP SCC & neg & $\mathrm{PTR} \pm \mathrm{BND}$ & pT4G3N0 \\
\hline 30 & OC SCC & - & $\mathrm{PTR} \pm \mathrm{BND}$ & pT4G3N1 \\
\hline 31 & OC SCC & - & $\mathrm{PTR} \pm \mathrm{BND}$ & pT4aG3N1 \\
\hline 32 & OC SCC & - & $\mathrm{PTR} \pm \mathrm{BND}$ & pT4G3N2c \\
\hline 33 & OC SCC & - & $\mathrm{PTR} \pm \mathrm{BND}$ & pT4G3N2b \\
\hline 34 & OC SCC & - & $\mathrm{PTR} \pm \mathrm{BND}$ & pT3G2N0 \\
\hline 35 & OC SCC & - & $\mathrm{PTR} \pm \mathrm{IND}$ & pT2G3N1 \\
\hline 36 & OC SCC & - & $\mathrm{PTR} \pm \mathrm{BND}$ & pT3G2N0 \\
\hline 37 & OC SCC & - & $\mathrm{PTR} \pm \mathrm{IND}$ & pT2G3N0 \\
\hline 38 & OC SCC & - & $\mathrm{PTR} \pm \mathrm{BND}$ & $\mathrm{pT} 1 \mathrm{G} 2 \mathrm{~N} 2 \mathrm{c}$ \\
\hline 39 & OC SCC & - & $\mathrm{PTR} \pm \mathrm{BND}$ & pT4aG3N1 \\
\hline 40 & OC SCC & - & $\mathrm{PTR} \pm \mathrm{BND}$ & pT4G3N2c \\
\hline
\end{tabular}

OC SCC: Oral cavity squamous-cell carcinoma; OP SCC: oropharynx squamous-cell carcinoma PTR: primary tumor resection; IND: ipsilateral neck dissection; BND: bilateral neck dissection; SLNB: sentinel lymph-node biopsy.

tumors under investigation. Immunohistochemical staining for HPV status was performed using the p16 ${ }^{\mathrm{INK}} 4 \mathrm{a}$ monoclonal antibody (E6H4, Roche Basel, Switzerland), in agreement with both manufacturer's recommendations as described previously (21). p16 IHC was scored as positive if there was strong, homogeneous and diffuse nuclear and cytoplasmic staining present in greater than $70 \%$ of the malignant cells. All other staining patterns were scored as negative.

CT protocol. All patients underwent CT scans of the head and neck, performed with a single multidetector CT scanner (Toshiba Multi-Slice Aquilion 64 system, Toshiba Medical Systems, Tokyo, Japan) after 

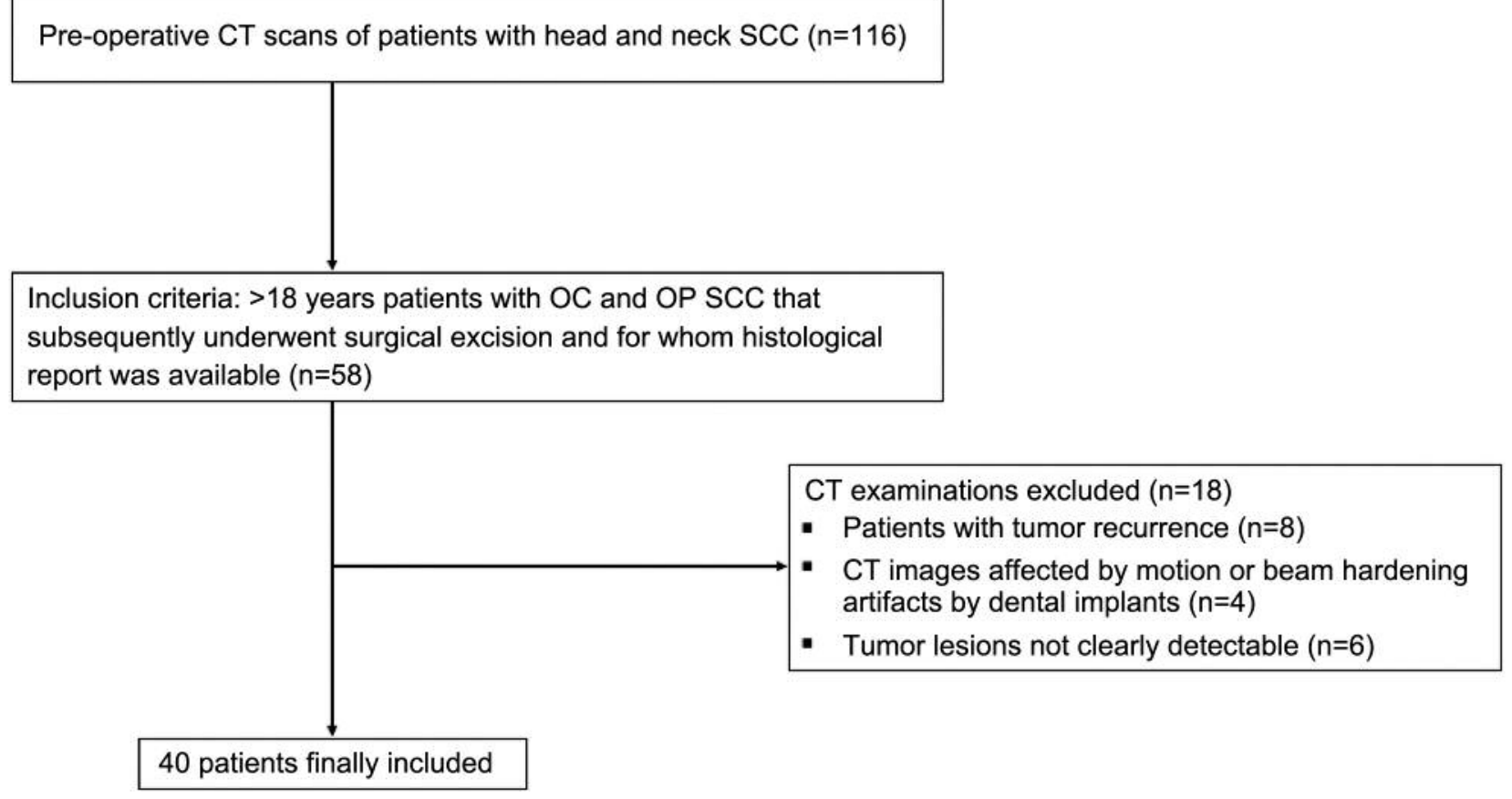

Figure 1. Flow-chart of patient selection. SCC: Squamous cell carcinoma, OC: oral cavity, OP: oropharynx.

intravenous injection of $100 \mathrm{ml}$ of nonionic iodinated contrast medium $\left[370 \mathrm{mg}\right.$ of iodine per milliliter (Iopamidol), Iopamiro ${ }^{\circledR} 370$; Bracco Imaging S.p.A.] at a flow rate of $3 \mathrm{ml} / \mathrm{s}$ with imaging delayed until 80 $\mathrm{s}$ after initiation of contrast medium injection. Scan parameters were $120 \mathrm{kV} ; 300 \mathrm{mAs}$; rotation time $=0.5 \mathrm{~s}$; pitch $=1$; collimation $=0.5 \mathrm{~mm}$ using a standard smoothing algorithm for axial image reconstruction; section thickness $=2.5 \mathrm{~mm}$; and intervals $=2.5 \mathrm{~mm}$. A $250 \times 250 \mathrm{~mm}$ field of view and $512 \times 512$ pixel matrix size were used for an average pixel size of approximately $0.5 \times 0.5 \mathrm{~mm}$ on the $\mathrm{x}$-axis and $\mathrm{y}$-axis and $2.5 \mathrm{~mm}$ on the $\mathrm{z}$-axis. Images were reviewed in soft-tissue algorithms.

Texture analysis. Lesion analysis was performed using an established image post-processing software $(22,23)$. CT images were imported on a dedicated software (ITK-SNAP 3.6.0) for tumor segmentation (24). Primary tumor lesions were identified on postcontrast CT images and manually segmented by drawing a polygonal ROI, further edited with paintbrush tools, excluding necrotic areas by a radiologist with 7 years' experience in head and neck imaging under the supervision of a senior radiologist with 20 years' experience in head and neck imaging. An example of ROI positioning is shown in Figure 3. ROIs were analyzed using a dedicated module on 3DSlicer, version 4.8 (heterogeneity CAD), extracting a total of 55 first, second and higher order texture-derived features (25). In particular, the second and last group were calculated from the Gray-Level Co-occurrence and Run Length Matrices. A graphical representation of the entire feature set, in the form of correlation matrix heatmaps for each classification endpoint, is shown in Figure 4. Then, prediction of tumor grade and nodal status in terms of presence/absence of cervical metastatic lymphnodes $(\mathrm{N} 0$ and $\mathrm{N}+)$ were tested $(26,27)$.
Machine learning analysis. In order to apply machine learning algorithms, the KNIME analytics platform (v. 3.7.1) was employed due to its widely acknowledged validity and the possible integration with other software (28-30). As some supervised algorithms can suffer from an unbalanced dataset, synthetic minority oversample technique (SMOTE) was first applied, determining an increase of the number of records till 50 in tumor grade and 42 in nodal status classification $(31,32)$. A hold-out was performed in order to split the population in a training $(75 \%)$ and a test set $(25 \%)$; the first one was used to learn models while the second one was an independent set of data to test the learned models (33). Then, the combination of a wrapper method and a 10-folds cross-validation were applied in order to find the best subset of features that made the accuracy the highest. Different algorithms were implemented:

- J48 is the Java implementation of the $\mathrm{c} 4.5$ decision tree, in which each node represents a test on an attribute, and each branch the results of the test and each leaf a class label (34);

- Multilayer perceptron (MLP) belongs to the category of neural networks, inspired by the composition of a biological neural network; its structure is made up of an input, hidden and an output layers, each with a different number of neurons (also defined as nodes); the training is conducted through the backpropagation technique (35);

- Naïve Bayes (NB) is a well-known algorithm based on the a priori probability of Bayes (36);

- K-Nearest Neighbor (KNN) is an instance-based classifier that assigns a label to each record taking into consideration the $k$ nearest neighbor elements; the commonly used distance is the linear Euclidean one (37). 

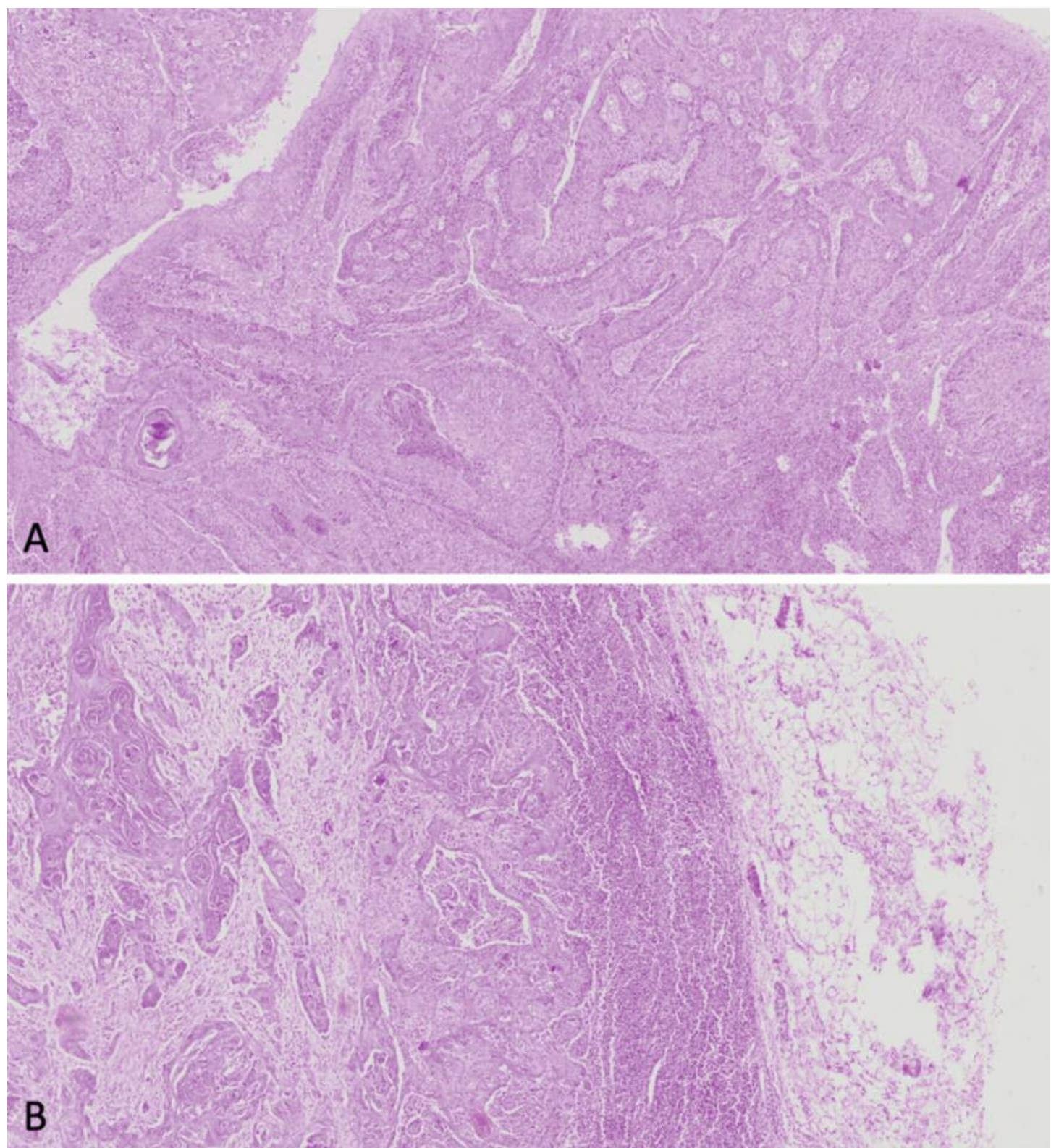

Figure 2. (A) Oral squamous cell carcinoma: H\&E-stained tissue sections demonstrated a poorly differentiated, infiltrating carcinoma (hematoxylineosin, original magnification $\times 40$ ). (B) A latero-cervical lymph node metastasis from the primary tumor in A (hematoxylin-eosin, original magnification $\times 40$ ).

Ensembles of the previous algorithms were also employed, particularly bagging and boosting. Bagging is useful to reduce variance, avoid overfitting of the model, improve the stability and accuracy of algorithms (38). The most famous boosting approach is called Adaboost and it converts weak learners into stronger ones through a target selection of features and a significant reduction of variance in supervised algorithms (39). A wide range of evaluation metrics is present in literature; in this study accuracy, sensitivity, specificity and area under the curve receiving operating characteristic (AUROC) were considered to compare the results (40). AUROC is a qualitative metric for binary classification between 0 and +1 where 0.5 represents the accuracy of a random guess.

Statistical analysis. Descriptive statistics were performed using the statistical package for social science (SPSS) software (version 24, IBM, Armonk, NY, USA). The normal distribution of quantitative TA features was assessed using the Shapiro-Wilk test. Accordingly, unpaired $t$-test and Mann Whitney- $U$-test were performed to compare each TA feature between G2 and G3 as well as between $\mathrm{N} 0$ and $\mathrm{N}+$ cases. A $p$-value $\leq 0.05$ was considered statistically significant. 

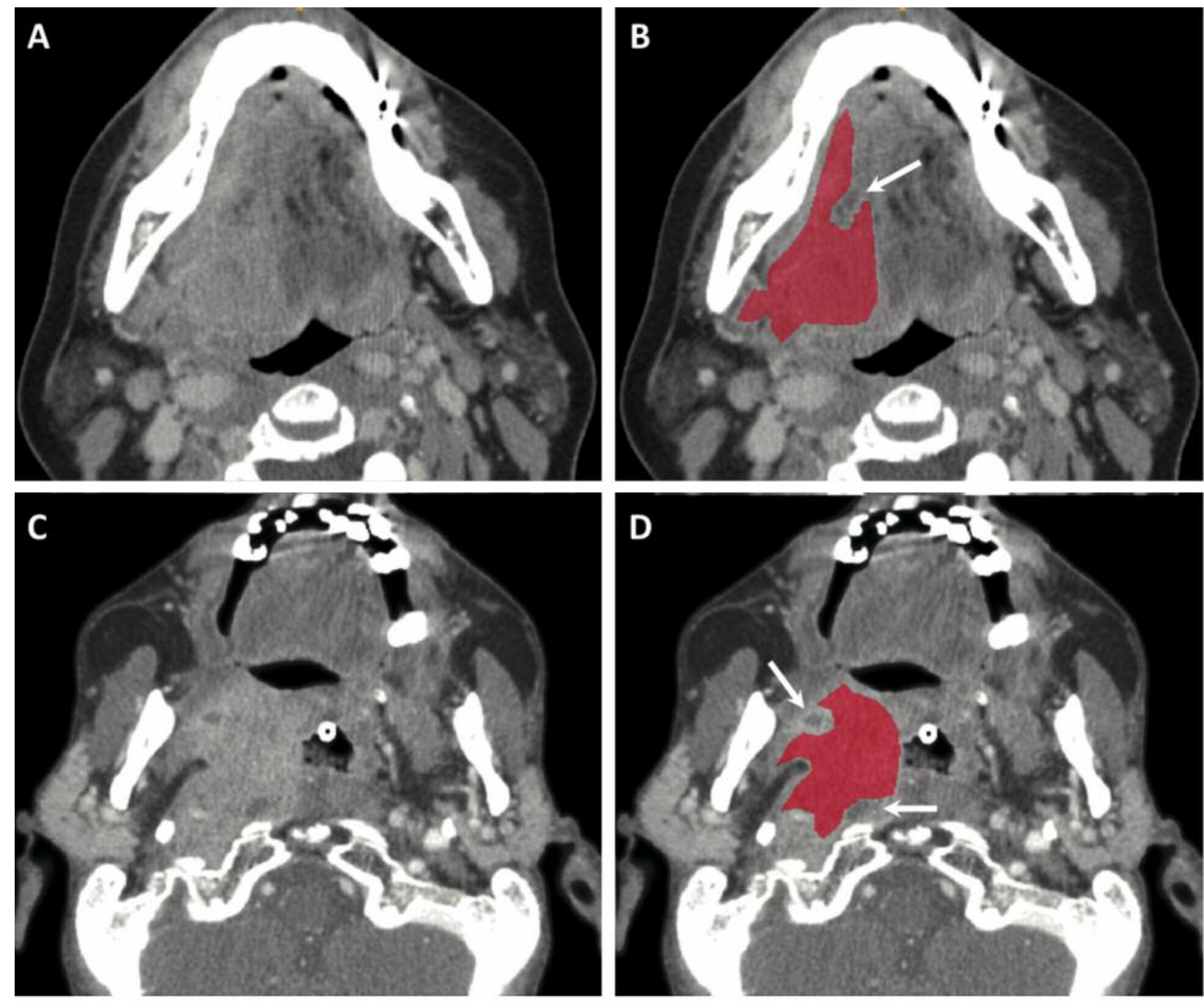

Figure 3. Examples of tumor segmentation. Axial contrast-enhanced CT images of oral cavity (A) and oropharynx (C) tumor lesions, along with the corresponding hand-drawn regions of interest (superimposed in red; $B, D$ ), positioned excluding necrotic areas (arrows).

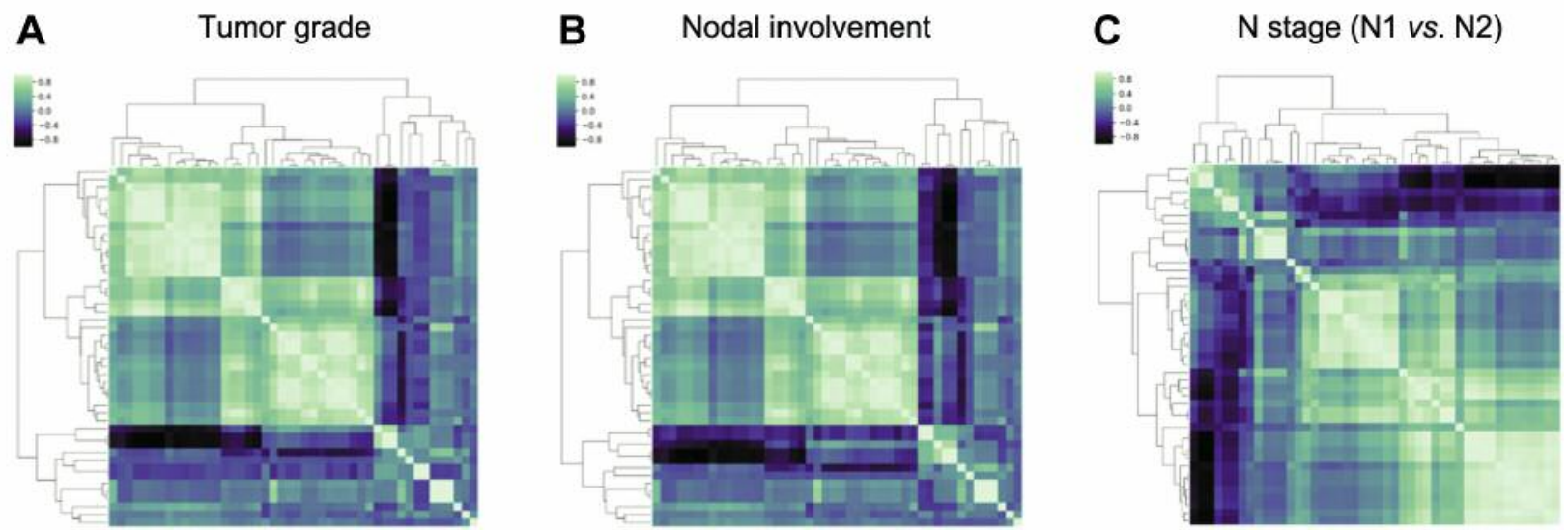

Figure 4. Feature correlation matrices represented as hierarchically-clustered heatmaps in relation to tumor grade prediction (A), nodal status presence $(B)$ and to $N$ stage definition $(C)$. 
Table II. Tumor grade classification.

\begin{tabular}{lccrrc}
\hline G2/G3 & Algorithms & Accuracy & Sensitivity & Specificity & AUCROC \\
\hline No ensemble & J48 & 78.6 & 85.7 & 71.4 & 0.888 \\
& MLP & 78.6 & 85.7 & 71.4 & 0.816 \\
& NB & 92.9 & 85.7 & 100.0 & 0.980 \\
& KNN & 78.6 & 71.4 & 85.7 & 0.878 \\
Bagging & J48 & 78.6 & 85.7 & 71.4 & 0.939 \\
& MLP & 71.4 & 85.7 & 57.1 & 0.852 \\
& NB & 92.9 & 85.7 & 100.0 & 0.857 \\
Adaboost & KNN & 92.9 & 100.0 & 85.7 & 0.939 \\
& J48 & 85.7 & 85.7 & 85.7 & 0.939 \\
& MLP & 85.7 & 71.4 & 100.0 & 0.878 \\
& NB & 78.6 & 85.7 & 71.4 & 0.827 \\
& KNN & 85.7 & 85.7 & 85.7 & 0.867 \\
\hline
\end{tabular}

MLP: Multilayer perception; NB: naïve Bayes; KNN: nearest neighbor; AUCROC: area under the curve receiving operating characteristic.

\section{Results}

Machine learning analysis. Performances of all algorithms for classification of tumor grade and nodal status are reported in Tables II and III, respectively. Regarding the classification of tumor grade, the best accuracy was achieved by NB and bagging of NB and KNN (92.9\%) while the highest AUROC was obtained by NB, bagging of J48 and KNN, boosting of J48 that were all over 0.900 . Concerning the classification of nodal status, J48 and NB, bagging of NB, boosting of J48 overcame the accuracy of $90.0 \%$ while the highest AUCROC was reached by $\mathrm{J} 48$ and $\mathrm{KNN}$ (both 0.900 ), bagging of J48 and KNN (respectively, 0.917 and 0.900 ), boosting of J48 and NB (0.900). Table IV shows all the features selected by the wrapper method per each algorithm, while Figure 5 summarizes the occurrence of the most frequent features (calculated over a total of 2 prediction and 12 algorithms).

Statistical evaluation. At unpaired $t$-test, short-run emphasis (SRE) was statistically different $(p=0.007)$ between G2 and G3 patients. SRE was also statistically different between N0 and $\mathrm{N}+$ patients along with Skewness $(p=0.05)$, and run_percentage (RP) $(p=0.01)$. At Mann-Whitney $U$-test a statistically significant difference was observed between $\mathrm{G} 2$ and G3 patients for Maximum_Intensity $(p=0.23)$, SRE (0.005) and long-run_low_gray-level_emphasis (LRLGLE) $(p=0.036)$ as well as between N0 and N1 patients in terms of Maximum_probability $(p=0.034)$ and RP $(p=0.023)$.

\section{Discussion}

According to our results, machine learning analysis applied to CT-derived TA features extracted from primary tumor lesions was useful to accurately predict tumor grade and
Table III. Nodal involvement classification.

\begin{tabular}{lccccc}
\hline N0/N1 & Algorithms & Accuracy & Sensitivity & Specificity & AUCROC \\
\hline No ensemble & J48 & 90.9 & 80.0 & 100.0 & 0.900 \\
& MLP & 72.7 & 60.0 & 83.3 & 0.800 \\
& NB & 90.9 & 100.0 & 83.3 & 0.867 \\
\multirow{5}{*}{ Bagging } & KNN & 81.8 & 100.0 & 66.7 & 0.900 \\
& J48 & 81.8 & 80.0 & 83.3 & 0.917 \\
& MLP & 72.7 & 100.0 & 50.0 & 0.833 \\
& NB & 92.9 & 85.7 & 100.0 & 0.857 \\
Adaboost & KNN & 72.7 & 100.0 & 50.0 & 0.900 \\
& J48 & 90.9 & 80.0 & 100.0 & 0.900 \\
& MLP & 72.7 & 80.0 & 66.7 & 0.650 \\
& NB & 81.8 & 60.0 & 100.0 & 0.900 \\
& KNN & 72.7 & 60.0 & 83.3 & 0.717 \\
\hline
\end{tabular}

MLP: Multilayer perception; NB: naïve Bayes; KNN: nearest neighbor; AUCROC: area under the curve receiving operating characteristic.

nodal status in patients with OC and OP SCC with a diagnostic accuracy of $92.9 \%$ and an overall good quality of the models, as proven by the high values of AUCROC. In predicting tumor grade, the application of boosting was successful not only with J48 and MLP (which is expected as they are unstable classifiers), but also with KNN which showed an improvement through the application of bagging, despite being a stable classifier. On the nodal status side, the application of ensemble methods was not successful as in the previous case. This issue is probably related to the small sample size; yet, they cannot lessen the overall high results obtained by this pilot investigation. Regarding TA features employed, those selected belonged to either first, second and higher order with the most frequently selected (i.e. SRE and LRLGLE) also resulting statistically different at descriptive statistic. Several studied previously investigated the usefulness of TA applied to CT images in predicting histological grade in solid tumors. Indeed, TA was found helpful in predicting tumor grade in lung adenocarcinoma and pancreatic neuroendocrine tumor (41-43). In detail, CT was useful for predicting grade $2 / 3$ pancreatic neuroendocrine tumors using not only imaging findings, but also CT TA features variables such as sphericity, skewness and kurtosis (42). Similarly, CT TA were found predictive of pancreatic neuroendocrine tumor aggressiveness and possibly useful to identify patients at risk of early disease progression after surgical resection (41). Furthermore, Liu et al. extracted TA features from contrast-enhanced CT images of patients with lung adenocarcinoma that were useful in predicting pathologic grade (43). Regarding the assessment of lymph node status, a $70 \%$ in diagnostic accuracy of classifying benign and malignant mediastinal lymph nodes in lung cancer patients has been described using TA features extracted by unenhanced CT images (44). Similarly, 
Table IV. Selected texture analysis features for each algorithm.

\begin{tabular}{|c|c|c|c|}
\hline & Algorithms & $\begin{array}{l}\text { Features in } \mathrm{G} \\
\text { classification }\end{array}$ & $\begin{array}{l}\text { Features in } \mathrm{N} \\
\text { classification }\end{array}$ \\
\hline \multirow[t]{4}{*}{ No ensemble } & $\mathrm{J} 48$ & $\begin{array}{l}\text { - Gray levels } \\
\text { - Median intensity } \\
\text { - Standard deviation } \\
\text { - Surface area } \mathrm{mm}^{2} \\
\text { - GLN }\end{array}$ & $\begin{array}{l}\text { - SRE } \\
\text { - LRLGLE }\end{array}$ \\
\hline & MLP & $\begin{array}{l}\text { - Energy } \\
\text { - Minimum intensity } \\
\text { - Skewness } \\
\text { - Autocorrelation } \\
\text { - Cluster prominence } \\
\text { - Variance GLCM } \\
\text { - SRE } \\
\text { - LRLGLE }\end{array}$ & $\begin{array}{l}\cdot \text { GLN } \\
\text { - LRLGLE }\end{array}$ \\
\hline & NB & $\begin{array}{l}\text { - Mean intensity } \\
\text { - Surface area } \mathrm{mm}^{2} \\
\text { - Sum variance } \\
\text { - SRE } \\
\text { - LRLGLE }\end{array}$ & $\begin{array}{l}\text { - Mean intensity } \\
\text { - Skewness } \\
\text { - Surface area } \mathrm{mm}^{2} \\
\text { - RP } \\
\text { - LRLGLE }\end{array}$ \\
\hline & $\mathrm{KNN}$ & - Mean intensity & $\begin{array}{l}\text { - Skewness } \\
\text { - Cluster shade } \\
\text { - SRE } \\
\text { - LGLRE }\end{array}$ \\
\hline \multirow[t]{4}{*}{ Bagging } & $\mathrm{J} 48$ & $\begin{array}{l}\text { - Median intensity } \\
\text { - Mean deviation } \\
\text { - Root mean square } \\
\text { - Kurtosis } \\
\text { - Homogeneity } 2 \\
\text { - Maximum probability } \\
\text { - LRHGLE }\end{array}$ & $\begin{array}{l}\text { - Range } \\
\text { - LRLGLE }\end{array}$ \\
\hline & MLP & $\begin{array}{l}\text { - Mean deviation } \\
\text { - Kurtosis } \\
\text { - Homogeneity2 } \\
\text { - IDMN } \\
\text { - SRE } \\
\text { - LRE } \\
\text { - LRHGLE }\end{array}$ & $\begin{array}{l}\text { - Skewness } \\
\text { - Cluster shade } \\
\text { - Contrast }\end{array}$ \\
\hline & NB & $\begin{array}{l}\text { - Entropy } \\
\text { - Root mean square } \\
\text { - Surface area } \mathrm{mm}^{2} \\
\text { - SRE } \\
\text { - RP } \\
\text { - LRLGL }\end{array}$ & $\begin{array}{l}\text { - Entropy } \\
\text { - Root mean square } \\
\text { - Surface area } \mathrm{mm}^{2} \\
\text { - SRE } \\
\text { - RP } \\
\text { - LRLGLE }\end{array}$ \\
\hline & $\mathrm{KNN}$ & $\begin{array}{l}\text { - Entropy } \\
\text { - Maximum intensity } \\
\text { - Surface area } \mathrm{mm}^{2} \\
\text { - SRE } \\
\text { - LRE } \\
\text { - LGLRE } \\
\text { - LRLGLE }\end{array}$ & $\begin{array}{l}\text { - Uniformity } \\
\text { - Cluster shade } \\
\text { - Contrast } \\
\text { - Sum variance } \\
\text { - LRLGLE }\end{array}$ \\
\hline
\end{tabular}

Andersen and colleagues found a statistically significant difference between CT TA features of benign and malignant lymph nodes in patients with non-small cells lung carcinoma (45). TA has been also applied to ultrasound images, showing a diagnostic accuracy of $98.8 \%$ in classifying

\begin{tabular}{|c|c|c|c|}
\hline & Algorithms & $\begin{array}{l}\text { Features in } \mathrm{G} \\
\text { classification }\end{array}$ & $\begin{array}{l}\text { Features in } \mathrm{N} \\
\text { classification }\end{array}$ \\
\hline \multirow[t]{4}{*}{ Adaboost } & $\mathrm{J} 48$ & $\begin{array}{l}\text { - Median intensity } \\
\text { - Root mean square } \\
\text { - Cluster shade } \\
\text { - SRE }\end{array}$ & $\begin{array}{l}\text { - Mean intensity } \\
\text { - Skewness } \\
\text { - Cluster tendency } \\
\text { - SRE } \\
\text { - LRLGLE }\end{array}$ \\
\hline & MLP & $\begin{array}{l}\text { - Mean intensity } \\
\text { - SRE } \\
\text { - RP } \\
\text { - LRLGLE }\end{array}$ & $\begin{array}{l}- \text { Kurtosis } \\
\text { - SRE } \\
\text { - RLN } \\
\text { - LRLGLE }\end{array}$ \\
\hline & NB & $\begin{array}{l}\text { - Root mean square } \\
\text { - Kurtosis } \\
\text { - Surface area mm }{ }^{2} \\
\text { - Homogeneity } 1 \\
\text { - Maximum probability } \\
\text { - SRE } \\
\text { - SRLGLE } \\
\text { - LRLGLE }\end{array}$ & $\begin{array}{l}\text { - Kurtosis } \\
\text { - SRE } \\
\text { - LRLGLE }\end{array}$ \\
\hline & $\mathrm{KNN}$ & $\begin{array}{l}\text { - Autocorrelation } \\
\text { - Cluster tendency } \\
\text { - Variance GLCM } \\
\text { - LRE } \\
\text { - HGLRE } \\
\text { - LRLGLE }\end{array}$ & $\begin{array}{l}\text { - Uniformity } \\
\text { - LRLGLE }\end{array}$ \\
\hline
\end{tabular}

MLP: Multilayer perceptron; NB: naïve Bayes; KNN: nearest neighbor; AUCROC: area under the curve receiving operating characteristic.

tumor-free and metastatic cervical lymph nodes in patients with papillary thyroid carcinoma (46). Differently from our study, these authors extracted TA features directly from detected lymph nodes on CT or ultrasound images. According to our experience, useful data on nodal status could be obtained even from the primary tumor mass, probably related to tumor heterogeneity and aggressiveness. Both tumor grade and nodal status are well recognized prognostic factors in terms of presence/absence of distant metastasis and overall survival in head and neck cancer patients. As a consequence, providing pre-treatment data concerning tumor grade and the possibility of lymph node status using a routinely used imaging technique may have a significant impact on therapeutic strategies and clinical outcome. Previous studies also analyzed the role of texture analysis applied to CT and MR imaging in head and neck cancer patients. Recently, first-order MR texture analysis applied to both primary tumor and lymph-nodes was found useful to predict extracapsular nodal spread in patients with OC SCC (47). Furthermore, TA has been previously applied on CT images to assess its reliability in predicting HPV status $(15,16)$. The authors found statistically significant differences in texture features between HPV positive and negative patients with OP tumors, thus suggesting this 


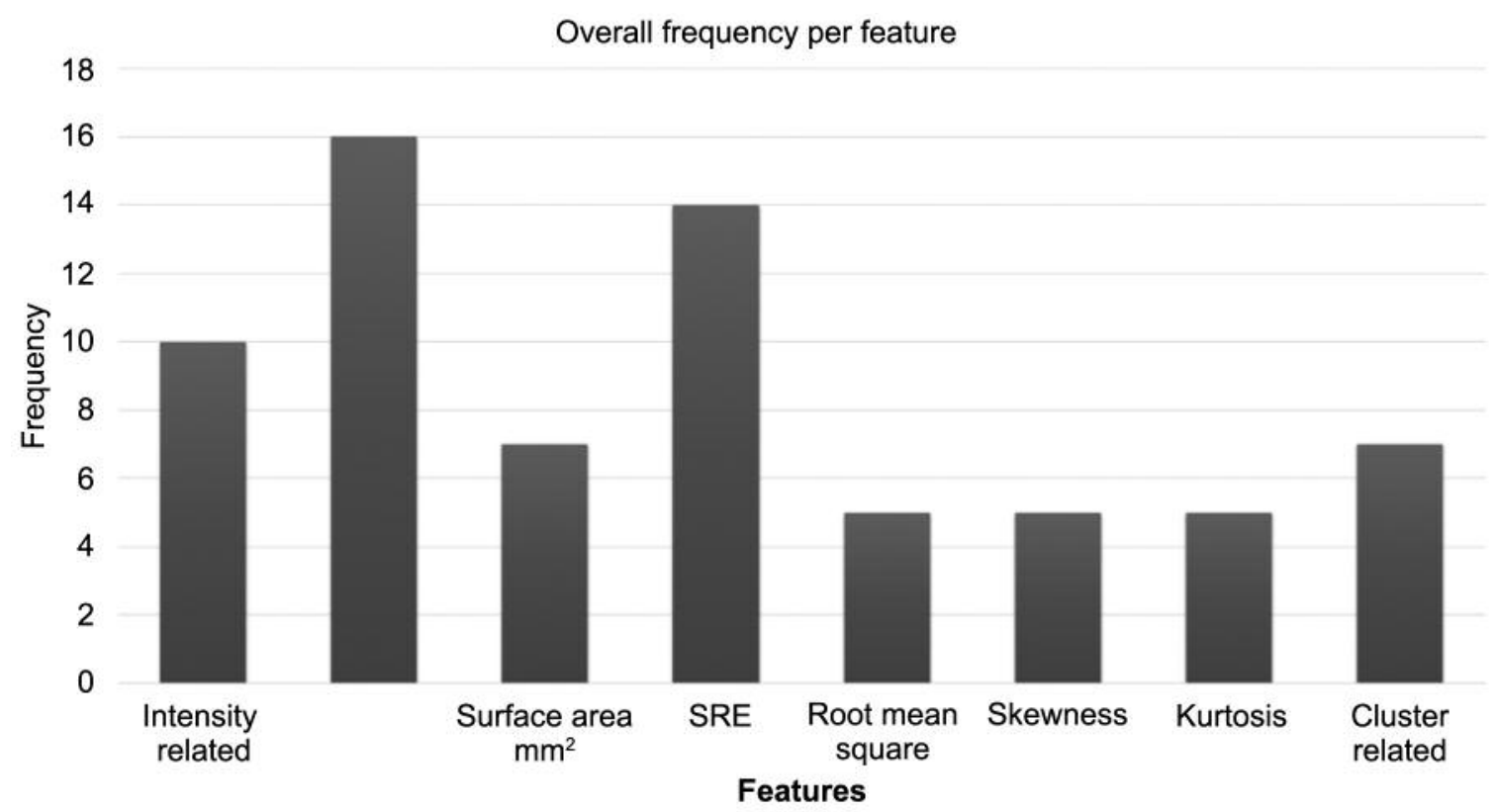

Figure 5. Histograms representing the most frequently selected features for both tumor grade and nodal status classifications. LRLGLE: Longrun_low_gray-level_emphasis; SRE: short-run_emphasis.

technique as an adjunct to the evaluation of HPV status. The potential of radiomics applied to PET-CT and CT images has been also reported in assessing the risk of specific tumor outcomes, in terms of locoregional recurrence and distant metastases (19). In a recent study, Dang and colleagues analyzed MR images of 16 patients with OP carcinoma using TA, showing a diagnostic accuracy of $80 \%$ in predicting $\mathrm{p} 53$ status (17). Regarding the employment of TA in predicting head and neck patients' outcome, pre-treatment CT images of 62 patients with head and neck cancer who underwent chemo-radiotherapy were analyzed in a recent study (18), showing that CT texture parameters were associated with local failure. Similarly, a population of 72 patients with head and neck carcinoma candidate to induction chemotherapy was evaluated using CT texture and histogram analysis of the primary tumor mass to predict the prognosis (20). The authors found that CT texture and histogram analysis parameters were associated with overall survival. To the best of our knowledge, this is the first study assessing tumor grade and lymph-node status in head and neck cancer patients using CT images. Our investigation expands the current knowledge about the use of TA and ML in head and neck cancer patients showing that such an approach, applied to primary tumor lesions, allows for accurate prediction not only of tumor grade but also of nodal status. A personalized, even more aggressive surgical approach could be, therefore, proposed. The major limitation of our study is the small sample size that: a) enabled us to prove the feasibility of many machine learning algorithms in this context without identifying one best algorithm; and b) did not allow to further stratify the $\mathrm{N}$ status and particularly to identify $\mathrm{N} 2 \mathrm{c}$ patients with contralateral metastatic lymph-nodes. Thus, future multicenter studies are strongly needed to confirm our preliminary findings.

In conclusion, ML analysis using contrast-enhanced CT TA applied to primary tumor lesions in patients with OC and OP may represent a reliable tool in predicting tumor grade and nodal status; it could also have a role in establishing the most appropriate surgical strategies and identifying high-risk patients.

\section{Conflicts of Interest}

The Authors declare that they have no conflicts of interest.

\section{Authors' Contributions}

Valeria Romeo: Study conception and design, acquisition, data analysis and interpretation, drafting of manuscript and critical revision; Renato Cuocolo: Acquisition, data analysis and interpretation, drafting of manuscript and critical revision; Carlo Ricciardi: Data acquisition, analysis and interpretation; Lorenzo Ugga: Data acquisition, analysis and interpretation; Sirio Ugga: Data analysis and interpretation, drafting of manuscript and critical revision; Francesco Verde: Data analysis and interpretation, critical revision; Arnaldo Stanzione: Data acquisition, analysis and interpretation; Daniela Russo: Data acquisition, analysis and interpretation; Giovanni Improta: Data 
analysis and interpretation, critical revision; Andrea Elefante: Data analysis and interpretation, drafting of manuscript and critical revision; Stefania Staibano: Study conception and design, critical revision; Arturo Brunetti: Study conception and design, critical revision.

\section{Acknowledgements}

The Author disclosed receipt of the following financial support for the research, authorship, and/or publication of this article: this work was supported by the project "Development of an integrated radiomic and phenotypic system for the diagnosis, prognosis and personalization of therapy of head and neck tumors. eMORFORAD technologic platform- Regione Campania”.

\section{References}

1 Ferlay J, Soerjomataram I, Dikshit R, Eser S, Mathers C, Rebelo M, Parkin DM, Forman D and Bray F: Cancer incidence and mortality worldwide: Sources, methods and major patterns in GLOBOCAN 2012. Int J Cancer, 2015. PMID: 25220842. DOI: 10.1002/ijc. 29210

2 Rettig EM and D'Souza G: Epidemiology of head and neck cancer. Surg Oncol Clin N Am 24: 379-396, 2015. PMID: 25979389. DOI: $10.1016 /$ j.soc.2015.03.001

3 Gregoire V, Lefebvre JL, Licitra L and Felip E: Squamous cell carcinoma of the head and neck: EHNS-ESMO-ESTRO Clinical Practice Guidelines for diagnosis, treatment and follow-up. Ann Oncol 21(Suppl 5): v184-186, 2010. PMID: 20555077. DOI: 10.1093/annonc/mdq185

4 Colevas AD, Yom SS, Pfister DG, Spencer S, Adelstein D, Adkins D, Brizel DM, Burtness B, Busse PM, Caudell JJ, Cmelak AJ, Eisele DW, Fenton M, Foote RL, Gilbert J, Gillison ML, Haddad RI, Hicks Jr. WL, Hitchcock YJ, Jimeno A, Leizman D, Maghami E, Mell LK, Mittal BB, Pinto HA, Ridge JA, Rocco J, Rodriguez CP, Shah JP, Weber RS, Witek M, Worden F, Zhen W, Burns JL and Darlow SD: NCCN Guidelines Insights: Head and Neck Cancers, Version 1.2018. J Natl Compr Canc Netw 16: 479-490, 2018. PMID: 29752322. DOI: 10.6004/jnccn.2018.0026

5 Jerjes W, Upile T, Petrie A, Riskalla A, Hamdoon Z, Vourvachis M, Karavidas K, Jay A, Sandison A, Thomas GJ, Kalavrezos N and Hopper C: Clinicopathological parameters, recurrence, locoregional and distant metastasis in $115 \mathrm{~T} 1-\mathrm{T} 2$ oral squamous cell carcinoma patients. Head Neck Oncol 2: 9, 2010. PMID: 20406474. DOI: $10.1186 / 1758-3284-2-9$

6 Kademani D, Bell RB, Bagheri S, Holmgren E, Dierks E, Potter $\mathrm{B}$ and Homer L: Prognostic factors in intraoral squamous cell carcinoma: The influence of histologic grade. J Oral Maxillofac Surg 63: 1599-1605, 2005. PMID: 16243176. DOI: 10.1016/j.joms.2005.07.011

7 Fortin A, Couture C, Doucet R, Albert M, Allard J and Tetu B: Does histologic grade have a role in the management of head and neck cancers? J Clin Oncol 19: 4107-4116, 2001. PMID: 11689578. DOI: 10.1200/JCO.2001.19.21.4107

8 Bisdas S, Baghi M, Smolarz A, Pihno NC, Lehnert T, Knecht R, Mack MG, Vogl TJ, Tuerkay S and Koh TS: Quantitative measurements of perfusion and permeability of oropharyngeal and oral cavity cancer, recurrent disease, and associated lymph nodes using first-pass contrast-enhanced computed tomography studies. Invest Radiol 42: 172-179, 2007. PMID: 17287647. DOI: 10.1097/01.rli.0000252496.74242.0b

9 Jin GQ, Yang J, Liu LD, Su DK, Wang DP, Zhao SF and Liao ZL: The diagnostic value of 1.5-T diffusion-weighted MR imaging in detecting 5 to $10 \mathrm{~mm}$ metastatic cervical lymph nodes of nasopharyngeal carcinoma. Med 95: e4286, 2016. PMID: 27512841. DOI: 10.1097/MD.0000000000004286

10 Trojanowska A, Trojanowski P, Bisdas S, Staskiewicz G, Drop A, Klatka J and Bobek-Billewicz B: Squamous cell cancer of hypopharynx and larynx - evaluation of metastatic nodal disease based on computed tomography perfusion studies. Eur J Radiol 81: 1034-1039, 2012. PMID: 21324623. DOI: 10.1016/j.ejrad. 2011.01.084

11 Choy G, Khalilzadeh O, Michalski M, Do S, Samir AE, Pianykh OS, Geis JR, Pandharipande PV, Brink JA and Dreyer KJ: Current applications and future impact of machine learning in radiology. Radiology 288: 318-328, 2018. PMID: 29944078. DOI: $10.1148 /$ radiol.2018171820

12 Gallo C, Ciavarella D, Santarelli A, Ranieri E, Colella G, Lo Muzio L and Lo Russo L: Potential salivary proteomic markers of oral squamous cell carcinoma. Cancer Genomics Proteomics 13: 55-61, 2016. PMID: 26708599.

13 Huang S, Nianguang CAI, Penzuti Pacheco P, Narandes S, Wang $\mathrm{Y}$ and Wayne XU: Applications of support vector machine (SVM) learning in cancer genomics. Cancer Genomics Proteomics 15: 41-51, 2018. PMID: 29275361. DOI: 10.21873/cgp.20063

14 Siristatidis C, Vogiatzi P, Pouliakis A, Trivella M, Papantoniou $\mathrm{N}$ and Bettocchi S: Predicting IVF Outcome: A proposed webbased system using artificial intelligence. In Vivo 4: 507-512, 2016. PMID: 27381616

15 Buch K, Fujita A, Li B, Kawashima Y, Qureshi MM and Sakai $\mathrm{O}$ : Using texture analysis to determine human papillomavirus status of oropharyngeal squamous cell carcinomas on CT. AJNR Am J Neuroradiol 36: 1343-1348, 2015. PMID: 25836725. DOI: 10.3174/ajnr.A4285

16 Fujita A, Buch K, Li B, Kawashima Y, Qureshi MM and Sakai O: Difference between HPV-positive and HPV-negative nonoropharyngeal head and neck cancer: Texture analysis features on CT. J Comput Assist Tomogr 40: 43-47, 2016. PMID: 26466116. DOI: $10.1097 /$ RCT.0000000000000320

17 Dang M, Lysack JT, Wu T, Matthews TW, Chandarana SP, Brockton NT, Bose P, Bansal G, Cheng H, Mitchell JR and Dort JC: MRI texture analysis predicts p53 status in head and neck squamous cell carcinoma. AJNR Am J Neuroradiol 36: 166-170, 2015. PMID: 25258367. DOI: 10.3174/ajnr.A4110

18 Kuno H, Qureshi MM, Chapman MN, Li B, Andreu-Arasa VC, Onoue K, Truong MT and Sakai O: CT texture analysis potentially predicts local failure in head and neck squamous cell carcinoma treated with chemoradiotherapy. AJNR Am J Neuroradiol 38: 2334-2340, 2017. PMID: 29025727. DOI: 10.3174/ajnr.A5407

19 Vallieres M, Kay-Rivest E, Perrin LJ, Liem X, Furstoss C, Aerts H, Khaouam N, Nguyen-Tan PF, Wang CS, Sultanem K, Seuntjens $\mathrm{J}$ and El Naqa I: Radiomics strategies for risk assessment of tumour failure in head-and-neck cancer. Sci Rep 7: 10117, 2017. PMID: 28860628. DOI: 10.1038/s41598-017-10371-5

20 Zhang H, Graham CM, Elci O, Griswold ME, Zhang X, Khan MA, Pitman K, Caudell JJ, Hamilton RD, Ganeshan B and Smith AD: Locally advanced squamous cell carcinoma of the head and neck: CT texture and histogram analysis allow 
independent prediction of overall survival in patients treated with induction chemotherapy. Radiology 269: 801-809, 2013. PMID: 23912620. DOI: 10.1148/radiol.13130110

21 Russo A and Russo G: Ribosomal proteins control or bypass p53 during nucleolar stress. Int J Mol Sci 18: 140, 2017. PMID: 28085118. DOI: $10.3390 /$ ijms 18010140

22 Romeo V, Maurea S, Cuocolo R, Petretta M, Mainenti PP, Verde F, Coppola M, Dell'Aversana S and Brunetti A: Characterization of adrenal lesions on unenhanced MRI using texture analysis: A machine-learning approach. J Magn Reson Imaging 48: 198-204, 2018. PMID: 29341325. DOI: 10.1002/jmri.25954

23 Cuocolo R, Stanzione A, Ponsiglione A, Romeo V, Verde F, Creta M, La Rocca R, Longo N, Pace L and Imbriaco M: Clinically significant prostate cancer detection on MRI: A radiomic shape features study. Eur J Radiol 116: 144-149, 2019. PMID: 31153556. DOI: 10.1016/j.ejrad.2019.05.006

24 Yushkevich PA, Piven J, Hazlett HC, Smith RG, Ho S, Gee JC and Gerig G: User-guided 3D active contour segmentation of anatomical structures: significantly improved efficiency and reliability. Neuroimage 31: 1116-1128, 2006. PMID: 16545965. DOI: $10.1016 /$ j.neuroimage.2006.01.015

25 Fedorov A, Beichel R, Kalpathy-Cramer J, Finet J, Fillion-Robin JC, Pujol S, Bauer C, Jennings D, Fennessy F, Sonka M, Buatti J, Aylward S, Miller J V, Pieper S and Kikinis R: 3D Slicer as an image computing platform for the Quantitative Imaging Network. Magn Reson Imaging 30: 1323-1341, 2012. PMID: 22770690. DOI: 10.1016/j.mri.2012.05.001

26 Chang P, Grinband J, Weinberg BD, Bardis M, Khy M, Cadena G, Su MY, Cha S, Filippi CG, Bota D, Baldi P, Poisson LM, Jain $\mathrm{R}$ and Chow D: Deep-learning convolutional neural networks accurately classify genetic mutations in gliomas. AJNR Am J Neuroradiol 39: 1201-1207, 2018. PMID: 29748206. DOI: 10.3174/ajnr.A5667

27 Taylor JC and Fenner JW: Comparison of machine learning and semi-quantification algorithms for (I123)FP-CIT classification: the beginning of the end for semi-quantification? EJNMMI Phys 4: 29, 2017. PMID: 29188397. DOI: 10.1186/s40658-017-0196-1

28 Berthold MR, Cebron N, Dill F, Gabriel TR, Kötter T, Meinl T, Ohl P, Thiel $\mathrm{K}$ and Wiswedel B: KNIME - the Konstanz information miner. ACM SIGKDD Explor Newsl 11: 26, 2009.

29 Warr WA: Scientific workflow systems: Pipeline Pilot and KNIME. J Comput Aided Mol Des 26: 801-804, 2012. PMID: 22644661. DOI: $10.1007 / \mathrm{s} 10822-012-9577-7$

30 Romeo V, Ricciardi C, Cuocolo R, Stanzione A, Verde F, Sarno L, Improta G, Mainenti PP, D'Armiento M, Brunetti A and Maurea S: Machine learning analysis of MRI-derived texture features to predict placenta accreta spectrum in patients with placenta previa. Magn Reson Imaging 64: 71-76, 2019. PMID: 31102613. DOI: 10.1016/j.mri.2019.05.017

31 Rastogi AK, Narang N and Siddiqui ZA: Imbalanced big data classification. In: ACM International Conference Proceeding Series, pp. 1-6, 2018.

32 Chawla NV, Bowyer KW, Hall LO and Kegelmeyer WP: SMOTE: Synthetic minority over-sampling technique. J Artif Intell Res 16: 321-357, 2002. PMID: 18190633. DOI: 10.1613/jair.953

33 Kohavi R: A study of cross-validation and bootstrap for accuracy estimation and model selection. Int Jt Conf Artif Intell 1995, 1995.

34 Quinlan JR: Induction of decision trees. Mach Learn 1: 81-106, 1986.
35 Pal SK and Mitra S: Multilayer Perceptron, fuzzy sets, and classification. IEEE Trans Neural Networks 3: 683-697, 1992. DOI: $10.1109 / 72.159058$

36 Jiang $\mathrm{L}$ and $\mathrm{Li} \mathrm{C}$ : Scaling up the accuracy of decision-tree classifiers: A Naive-Bayes combination. J Comput 6: 1325-1331, 2011. DOI: $10.4304 /$ jcp.6.7.1325-1331

37 Cover T and Hart P: Nearest neighbor pattern classification. IEEE Trans Inf Theory 13: 21-27, 1967. DOI: 10.1109/TIT.1967. 1053964

38 Breiman L: Bagging predictors. Mach Learn 24: 123-140, 2004. DOI: $10.1023 / \mathrm{A}: 1018054314350$

39 Freund Y and Schapire RE: Experiments with a new boosting algorithm. ICML 1996, 1996.

40 Hossin M and Sulaiman MN: A review on evaluation metrics for data classification evaluations. Int J Data Min Knowl Manag Process 5: 1-11, 2015. DOI: 10.5121/ijdkp.2015.5201

41 Canellas R, Burk KS, Parakh A and Sahani DV: Prediction of pancreatic neuroendocrine tumor grade based on CT features and texture analysis. AJR Am J Roentgenol 210: 341-346, 2018. PMID: 29140113. DOI: 10.2214/AJR.17.18417

42 Choi TW, Kim JH, Yu MH, Park SJ and Han JK: Pancreatic neuroendocrine tumor: prediction of the tumor grade using CT findings and computerized texture analysis. Acta Radiol 59: 383392, 2018. PMID: 28766979. DOI: 10.1177/0284185117725367

43 Liu Y, Liu S, Qu F, Li Q, Cheng R and Ye Z: Tumor heterogeneity assessed by texture analysis on contrast-enhanced $\mathrm{CT}$ in lung adenocarcinoma: association with pathologic grade. Oncotarget 8 : 53664-53674, 2017. PMID: 28881840. DOI: 10.18632/oncotarget. 15399

44 Pham TD, Watanabe Y, Higuchi M and Suzuki H: Texture analysis and synthesis of malignant and benign mediastinal lymph nodes in patients with lung cancer on computed tomography. Sci Rep 7: 43209, 2017. PMID: 28233795. DOI: 10.1038/srep43209

45 Andersen MB, Harders SW, Ganeshan B, Thygesen J, Torp Madsen $\mathrm{HH}$ and Rasmussen F: CT texture analysis can help differentiate between malignant and benign lymph nodes in the mediastinum in patients suspected for lung cancer. Acta radiol 57: 669-676, 2016. PMID: 26271125. DOI: 10.1177/0284185115598808

46 Ardakani AA, Rasekhi A, Mohammadi A, Motevalian E and Najafabad BK: Differentiation between metastatic and tumour-free cervical lymph nodes in patients with papillary thyroid carcinoma by grey-scale sonographic texture analysis. Polish J Radiol 83: 3746, 2018. PMID: 30038677. DOI: 10.5114/pjr.2018.75017

47 Frood R, Palkhi E, Barnfield M, Prestwich R, Vaidyanathan S and Scarsbrook A: Can MR textural analysis improve the prediction of extracapsular nodal spread in patients with oral cavity cancer? Eur Radiol 12: 5010-5018, 2018. PMID: 29872911. DOI: $10.1007 /$ s00330-018-5524-x 